\section{Structural myocardial involvement in adult patients with type 1 myotonic dystrophy}

\author{
Upinder K. Dhand,1 Faisal Raja,1 \\ Kul Aggarwal2 \\ Departments of 1 Neurology and \\ 2Cardiology, University of Missouri, \\ Columbia MO, USA
}

\section{Abstract \\ Myotonic dystrophy type 1 (DM1) is the} commonest muscular dystrophy in adults, affecting multiple organs in addition to skeletal muscles. Cardiac conduction system abnormalities are well recognized as an important component of DM1 phenotype; however, primary structural myocardial abnormalities, which may predispose these patients to congestive heart failure, are not as well characterized. We reviewed the retrospective analysis of the clinical and echocardiographic findings in adult patients with DM1. Among 27 patients (16 male; age 19-61 years) with DM1, the echocardiogram (ECHO) was abnormal in 10 (37\%) including one of 6 patients (16\%) with congenital myotonic dystrophy. Reduced left ventricular ejection fraction (LVEF $\leq 50 \%$ ) was noted in 5, diastolic dysfunction in 4, left atrial dilatation in 3 , left ventricular hypertrophy in 2 , apical hypokinesia in 1 and mitral valve prolapse in 3 patients. One patient had paradoxical septal movement in the setting of left bundle branch block. Echocardiographic abnormalities significantly correlated with older age; however, patients with systolic dysfunction on echocardiogram ranged in age from 27 to 52 years including 2 patients aged 27 and 34 years. We can conclude that echocardiographic abnormalities are frequent in adult patients with DM1. The incidence is similar in the classical and congenital type of DM1. Overall, echocardiographic abnormalities in DM1 correlate with increasing age; however, reduced LVEF is observed even at young age. Cardiac assessment and monitoring in adult patients with DM1 should include evaluation for primary myocardial involvement.

\section{Introduction}

Myotonic muscular dystrophy type 1 (DM1) is an autosomal dominant, multisystem degenerative disorder due to unstable expansion of CTG trinucleotide repeats.1,2 In addition to the clinical features of muscle wasting, weakness and myotonia, the patients with DM1 often have involvement of heart, eye, brain, endocrine and other organ systems, which impact the function, quality of life and survival in these patients. ${ }^{3-5}$ Cardiac involvement in the form of arrhythmias and/or conduction defects is frequent, occurring in $50-65 \%$ of patients with DM1.2-7 Primary cardiomyopathy in DM1 presenting as congestive heart failure is rare; however, subclinical systolic dysfunction has been reported with a prevalence of $0-28 \%$ in different studies using echocardiographic or magnetic resonance imaging. ${ }^{7-11}$ The frequency of cardiac conduction defects and tachy/bradyarrhythmias correlates significantly with increasing age, male gender, number of CTG repeats and neuromuscular disability.2,5,6,10,12 In contrast, there is no consistent association of such risk factors with structural myocardial involvement and systolic dysfunction. ${ }^{9,11,13}$ In the present study, we describe the nature and prevalence of various structural myocardial abnormalities as noted on echocardiographic imaging in adult patients with DM1, and attempt to define factors associated with the abnormal ECHO findings.

\section{Materials and Methods}

We retrospectively reviewed the clinical, electrocardiographic (EKG) and ECHO findings in adult patients with DM1. The information was obtained from the MDA (Muscular Dystrophy Association) clinic and database of the Echocardiography laboratory at our institute. The diagnosis of DM1 was based on characteristic clinical phenotype, positive family history consistent with autosomal dominant transmission, electromyographic findings, and/or expansion of CTG repeats on genetic testing. Adult patients ( $>18$ years), in whom echocardiogram results were available, were included in the study. Patients with known underlying cardiac disease (ischemic, congenital or valvular) were excluded. The medical records of the patients were reviewed for the demographics, history of cardiac symptoms, duration of illness, proximal (shoulder abduction, hip flexion) and distal (grip, ankle dorsiflexion) muscle strength in extremities [grade $0-5$ of Medical Research Council (MRC) scale],14 presence of congenital myotonic dystrophy, EKG findings, and number of CTG repeats if the results of genetic testing were known.

ECHO reports and video recordings (when available) were reviewed by a cardiologist (KA). Various parameters assessed on echocardiogram included left ventricular ejection fraction (LVEF), chamber enlargement, valvular dysfunction, wall motion abnormality and Doppler findings ( $\mathrm{E} / \mathrm{A}$ and $\mathrm{E}$ deceleration Time pulse wave recording from left ventricular inflow, Tricuspid Regurgitation maximum velocity on
Correspondence: Upinder K. Dhand, Department of Medicine, Division of Neurology, University of Tennessee Graduate School of Medicine, 1924 Alcoa Highway \#U114, Knoxville TN 37920, USA. Tel. +1.865.3059340 - Fax: +1.865 .3059144$

E-mail: udhand@mc.utmck.edu

Key words: cardiac involvement, echocardiogram, myopathy, myotonic dystrophy.

Contributions: the authors contributed equally.

Conflict of interest: the authors declare no potential conflict of interests.

Received for publication: 9 December 2012. Revision received: 23 February 2013.

Accepted for publication: 25 February 2013.

This work is licensed under a Creative Commons Attribution NonCommercial 3.0 License (CC BYNC 3.0).

(C) Copyright U.K. Dhand et al., 2013

Licensee PAGEPress, Italy

Neurology International 2013; 4:e5

doi:10.4081/ni.2013.e5

continuous wave Doppler). LVEF was calculated as: end diastolic volume-end systolic volume, divided by end diastolic volume. Patients with abnormal vs. normal ECHO were compared for age, gender, cardiac symptoms, EKG findings of prolonged PR interval, left anterior fascicular block and QTc interval, presence of congenital myotonic dystrophy (CMD), severe distal weakness (MRC <4), and presence of proximal muscle weakness $(\mathrm{MRC}<5)$.

Statistical analysis was performed by independent $\mathrm{t}$-test and Fisher exact test, and $\mathrm{P}$ value $<0.05$ was considered significant. The study was approved by the Institutional Review Board of the University of Missouri, USA.

\section{Results}

Among the 34 patients with DM1 in whom ECHO reports and/or recordings were available, 3 patients were excluded because of incomplete clinical information, and another 4 patients were excluded because of underlying cardiovascular disorder (2 with coronary artery disease, 1 with patent ductus arteriosus and 1 with acute pulmonary embolism at the time of ECHO). The remaining 27 patients (60\% men) aged $39.1 \pm 14.1$ years (range $19-61$ years) comprised the study group. History of cardiac symptoms (tachycardia, palpitations, or known atrial fibrillation) was present in 8 patients, proximal weakness was noted in 19 patients, and distal weakness was observed in 26 patients including severe $(\mathrm{MRC}<4)$ weak- 
ness in 13 patients. Six patients (age 19-54 years) had diagnosis of congenital myotonic dystrophy.

EKG was available in 25 patients and showed atrial flutter and atrial fibrillation in 1 patient each, first degree atrio-ventricular block (AVB1) in 8 patients, left anterior fascicular block in 7 patients, left bundle branch block (LBBB) and trifascicular block in 1 patient each. Results of genetic testing were available only in 9 patients (CTG repeats n 992600 ) and were not included in further statistical analysis.

An echocardiographic study was considered abnormal if one or more of the following were present: LVEF less than or equal to $50 \%$, regional wall motion abnormality, left atrial enlargement, moderate or severe valvular regurgitation, valvular stenosis, pulmonary hypertension or Doppler evidence of diastolic dysfunction or other significant finding not seen on a normal study. 15

Ten patients (37\%) had abnormal echocardiogram, including 5 patients with abnormality in more than one parameter. Reduced LVEF (range 35-50\%) was observed in $5(18.5 \%)$ patients, left atrial dilatation in 3 , left ventricular hypertrophy in 2 , and apical wall hypokinesia and paradoxical septal motion in 1 patient each. Mild diastolic dysfunction was noted in 4 patients. Two of these patients had evidence of impaired relaxation and 2 had pseudo-normalization pattern. Diastolic dysfunction on doppler study was the only abnormal finding in 2 patients. The patient with paradoxical septal movement had left bundle branch block on EKG and no other ECHO abnormality. Mitral valve prolapse (MVP) was noted in 3 patients, being an isolated finding in 2 patients, and in one patient it was associated with moderate mitral valve regurgitation and diastolic dysfunction.

Various clinical and EKG findings in patients with normal or abnormal ECHO are compared in Table 1. The presence of an abnormal ECHO significantly correlated only with older age $(\mathrm{P}=0.017)$. There was a trend for association with first degree atrio-ventricular block (AVB1), but this association was not statistically significant.

All 5 patients with systolic dysfunction (LVEF 35-50\%) were men, aged 27-52 years, who had DM1 for 6 to 27 years. The systolic dysfunction was mild in 2 (LVEF 50\%) and moderate (LVEF $35 \%, 37 \%$ and $40 \%$ ) in 3 patients. None of these patients had findings of dilated left ventricle. Clinical findings of congestive heart failure were not observed in any of these patients. Proximal muscle weakness was present in 4 patients, severe distal weakness in 3 and history of tachyarrhythmia in another 2 patients (Table 2). Any association between the subclinical systolic dysfunction and demographic or other risk factors, or
CTG repeat length could not be determined due to the small number of patients.

Among the 6 patients ( 2 female) with CMD, an abnormal ECHO was observed only in one patient ( 27 years-male) in the form of apical wall hypokinesia and LVEF $50 \%$. The observed frequency of abnormal ECHO in the congenital type was not different from the adult type of DM1 (Table 1).

\section{Discussion}

The significant findings in our study were frequent echocardiographic abnormalities in adult patients with DM1, with overall significant correlation with increasing age, but the subclinical systolic dysfunction (reduced LVEF) was observed even at younger $(<40$ year) age. We noted echocardiographic abnormalities in over one third of the adult patients with DM1, including findings of reduced LVEF in half of these patients. Other ECHO abnormalities included diastolic dysfunction, left atrial dilatation, mitral valve prolapse, regional wall motion abnormality and left ventricular hypertrophy. The pattern and prevalence of various abnormalities were similar to those observed by previous investigators. ${ }^{9-11,13}$ Such wide range of abnormalities appear to be commensurate with the reported pathological changes in DM1, of widespread patchy fibrosis and fatty degeneration affecting myocardium in all chambers. $1,3,16$

The overall echocardiographic abnormalities in our study correlated significantly with older age; however, 2 of the 5 patients with reduced LVEF were aged 27 and 34 years. In contrast, previous studies in a large cohort have documented significant association of systolic dysfunction in DM1 with increasing age, noting that it almost always occurred after the age of 40 years..$^{9,13}$ It is possible that mechanisms other than age related changes may also underlie the left ventricular dysfunc-

Table 1. Comparison of clinical and electrocardiographic findings in myotonic dystrophy type 1 patients with normal and abnormal echocardiogram.

\begin{tabular}{lccc} 
Parameter & $\begin{array}{c}\text { Total } \\
(\mathrm{n}=27)\end{array}$ & $\begin{array}{c}\text { Normal ECHO } \\
(\mathrm{n}=17)\end{array}$ & $\begin{array}{c}\text { Abnormal ECHO } \\
(\mathrm{n}=10)\end{array}$ \\
Age (years) & $39.1 \pm 14.1$ & $47.4 \pm 11.10$ & $34.2 \pm 13.6^{*}$ \\
Male & 16 & 9 & 7 \\
\hline Female & 11 & 8 & 3 \\
Duration of illness (years) & $21.0 \pm 13.3$ & $17.4 \pm 2.8$ & $25.9 \pm 13.2$ \\
\hline Cardiac symptoms & 8 & 5 & 3 \\
PR interval >200 ms & 8 & 2 & 6 \\
\hline Left anterior fascicular block & 7 & 5 & 2 \\
QTc ms & $414.6 \pm 30.1$ & $414.0 \pm 27.8$ & $415.5 \pm 34.7$ \\
\hline Proximal weakness & 19 & 11 & 8 \\
Severe distal weakness (MRC $<4)$ & 13 & 7 & 6 \\
\hline Congenital myotonic dystrophy & 6 & 5 & 1
\end{tabular}

Table 2. Characteristics of adult myotonic dystrophy type 1 patients with systolic dysfunction.

\begin{tabular}{|c|c|c|c|c|c|c|}
\hline N. & LVEF (\%) & $\begin{array}{c}\text { Age } \\
\text { (years) }\end{array}$ & $\begin{array}{l}\text { Duration } \\
\text { illness } \\
\text { (years) }\end{array}$ & $\begin{array}{c}\text { Other } \\
\text { ECHO } \\
\text { abnormality }\end{array}$ & EKG & $\begin{array}{c}\text { Weakness } \\
\text { proximal/distal } \\
<4\end{array}$ \\
\hline 1 & 50 & 40 & 11 & None & Normal & $\mathrm{Y} / \mathrm{Y}$ \\
\hline 16 & 37 & 34 & 9 & None & A Fib & $\mathrm{N} / \mathrm{N}$ \\
\hline 19 & 50 & 27 & $27^{*}$ & $\begin{array}{c}\text { Apical } \\
\text { hypokinesia }\end{array}$ & LAFB & $\mathrm{Y} / \mathrm{N}$ \\
\hline 23 & 35 & 52 & 27 & None & AVB1 & $\mathrm{Y} / \mathrm{Y}$ \\
\hline 28 & 40 & 47 & 6 & $\begin{array}{l}\text { Left atrial } \\
\text { dilatation }\end{array}$ & $\begin{array}{l}\text { AFL } \\
\text { AVB1 }\end{array}$ & $\mathrm{Y} /$ \\
\hline
\end{tabular}

*Congenital myotonic dystrophy. LVEF, left ventricular ejection fraction; ECHO, echocardiogram; EKG, electrocardiogram; AVB1, first degree atrioventricular block; LAFB, left anterior fascicular block; AFL, atrial flutter; A Fib, atrial fibrillation. 
tion in DM1 patients. Among other clinical factors evaluated to determine the DM1 patients at risk for left ventricular dysfunction, significantly higher incidence has been reported in male patients, ${ }^{11,13}$ but no consistent association has been observed with number of CTG repeats, duration of illness or neuromuscular disability.9-11,13 of note, all patients with reduced LVEF in our study were male; however, a specific relationship with various clinical and demographic factors could not be determined due to the small number of cases.

We observed mild diastolic dysfunction in 4 patients, and this was an isolated finding in 2 of these patients. Several studies, using conventional or tissue Doppler imaging have documented diastolic dysfunction in DM1 patients with normal ejection fraction, and hence these findings have been considered an early indicator of left ventricular dysfunction. ${ }^{17,18}$ It has been proposed that the myocardial degenerative process as well as poor relaxation due to myotonia may contribute to the diastolic dysfunction. $1,3,17$

Regional wall motion abnormality on ECHO has been reported in 11.2-28\% patients with DM1.9,10 We noted this finding only in one patient in the form of apical hypokinesia. An interesting observation was paradoxical movement of the interventricular septum in a patient who also had left bundle branch block (LBBB). In other cardiac conditions, LBBB is reported to be associated with paradoxical septal movement and may have significant hemodynamic consequences. ${ }^{19,20}$ However, paradoxical septal motion in the setting of LBBB has not been described previously in patients with DM1. Among other EKG changes, we noted a trend, albeit not significant, for association of prolonged PR interval with abnormal ECHO. Previous investigators have shown significant correlation of prolonged PR interval and/or prolonged QRS with reduced LVEF and regional wall motion abnormality in patients with DM1.5,10,13 On the other hand, Kaminski et al. ${ }^{12}$ observed that an abnormal ECHO in DM1 patients was a predictor of high risk for cardiac arrhythmias and conduction defects. Similarly to other investigators, we also found an interdependence of EKG and ECHO abnormalities in patients with DM1, such that the abnormality in either one could prompt further evaluation to determine the full range of cardiac involvement.

The findings of subclinical left ventricular dysfunction in DM1 patients in our study thus ranged from reduced LVEF to diastolic dysfunction and regional wall motion abnormality similar to the previous studies.9,10,12,13 Our study did not include a longitudinal follow up, therefore long term clinical impact of these ECHO changes could not be determined. Long term follow up of DM1 patients in a few stud- ies has revealed increasing frequency of left ventricular dysfunction, and association of ECHO abnormalities with increased mortality and sudden death. $5,10,13$

Congenital myotonic dystrophy is considered the severest phenotype of DM1, with high mortality in infancy and childhood, and only about $50 \%$ of patients survive to adulthood. ${ }^{21}$ In addition to cardiac conduction defects, obstructive cardiomyopathy and systolic dysfunction have been reported. ${ }^{22,23}$ In our study, abnormal ECHO (apical hypokinesia and LVEF 50\%) was noted in only 1 out of 6 patients with CMD. The incidence is similar to the adult type of DM1, and patients with CMD who survive to adulthood do not seem to have any increased vulnerability to myocardial involvement.

The major limitation of our study is the retrospective review of a relatively small number of patients, and genetic testing not being available in all patients. Therefore a definitive relationship of clinical features, EKG changes and CTG repeat length with structural myocardial involvement could not be assessed.

\section{Conclusions}

In conclusion, we found a wide range of echocardiographic abnormalities in over one third of adult patients with DM1. Subclinical systolic dysfunction on ECHO was present even at younger age ( $<40$ years), although myocardial involvement was more frequent with increasing age. The frequency of abnormal ECHO in adult patients with CMD was similar to that observed in patients with the classical type of DM1. The study also points to an interdependence of EKG and ECHO abnormalities. Our findings support the need for investigation of structural myocardial involvement in adult patients with DM1.

\section{References}

1. Phillips MF, Harper PS. Cardiac disease in myotonic dystrophy. Cardiovasc Res 1997;33:13-22.

2. Antonini G, Giubilei F, Mammarella A, et al. Natural history of cardiac involvement in myotonic dystrophy: correlation with CTG repeats. Neurology 2000;55:1207-9.

3. Pelargonio G, Dello Russo A, Sanna T, et al. Myotonic dystrophy and the heart. Heart 2002;88:665-70.

4. Mammarella A, Paradiso M, Antonini G, et al. Natural history of cardiac involvement in myotonic dystrophy (Steinert's disease): a 13-year follow-up study. Adv Ther 2000;17:238-51.
5. Groh WJ, Groh MR, Saha C, et al. Electrocardiographic abnormalities and sudden death in myotonic dystrophy type 1. N Engl J Med 2008;358:2688-97.

6. Cudia P, Bernasconi P, Chiodelli R, et al. Risk of arrhythmia in type I myotonic dystrophy: the role of clinical and genetic variables. J Neurol Neurosurg Psychiatry 2009; 80:790-3.

7. Petri H, Vissing J, Witting N, et al. Cardiac manifestations of myotonic dystrophy type 1. Int J Cardiol 2012;160:82-8.

8. De Ambroggi L, Raisaro A, Marchianó V, et al. Cardiac involvement in patients with myotonic dystrophy: characteristic features of magnetic resonance imaging. Eur Heart J 1995;16:1007-10.

9. Bhakta D, Lowe MR, Groh WJ. The prevalence of structural heart abnormalities in myotonic dystrophy type 1 . Am Heart J 2004;147:224-7.

10. Tokgozoglu LS, Ashizawa T, Pacifico A, et al. Cardiac involvement in a large kindred with myotonic dystrophy. Quantitative assessment and relation to size of CTG repeat expansion. JAMA 1995;274:813-9.

11. Hermans MC, Faber CG, Bekkers SC, et al. Structural and functional cardiac changes in myotonic dystrophy type 1: a cardiovascular magnetic resonance study. J Cardiovasc Magn Reson 2012;14:48.

12. Kaminsky P, Poussel M, Pruna L, et al. Organ dysfunction and muscular disability in myotonic dystrophy type 1 . Medicine (Baltimore) 2011;90:262-8.

13. Bhakta D, Groh MR, Shen C, et al. Increased mortality with left ventricular systolic dysfunction and heart failure in adults with myotonic dystrophy type 1 . Am Heart J 2010;160:1137-41.

14. Medical Research Council. Aids to examination of the peripheral nervous system. Memorandum No. 45. London: Her Majesty's Stationary office; 1976.

15. Lang RM, Bierig M, Devereux RB, et al. Chamber Quantification Writing Group; American Society of Echocardiography's Guidelines and Standards Committee; European Association of Echocardio-graphy. J Am Soc Echocardiogr 2005;18:144063.

16. Nguyen HH, Wolfe JT III, Holmes DR Jr, Edwards WD. Pathology of the cardiac conduction system in myotonic dystrophy: a study of 12 cases. J Am Coll Cardiol 1988; 11:662-71.

17. Fragola PV, Calo L, Luzi M, et al. Doppler echocardiographic assessment of left ventricular diastolic function in myotonic dystrophy. Cardiology 1997;88:498-502.

18. Vinereanu D, Bajaj BP, Fenton-May J, et al. Subclinical cardiac involvement in myoto-nic dystrophy manifesting as decreased myocardial Doppler velocities. 
Neuromu-scul Disord 2004;14:188-94.

19. Abbasi AS, Eber LM, MacAlpin RN, Kattus AA. Paradoxical motion of interventricular septum in left bundle branch block. Circulation 1974;49:423-7.

20. Fujii B, Takami M. Normalization of left ventricular function following cardiac resynchronization therapy: left bundle branch block as a potential etiology of dilated cardiomyopathy. Circ J 2008;72: 1030-3.

21. Reardon W, Newcombe R, Fenton I, et al. The natural history of congenital myotonic dystrophy: mortality and long term clinical aspects. Arch Dis Child 1993; 68:177-181.
22. Forsberg H, Olofsson B0, Eriksson A, Andersson S. Cardiac involvement in congenital myotonic dystrophy. Br Heart J 1990;63:119-21.

23. Muntoni F. Cardiac complications of childhood myopathies. J Child Neurol 2003;18: 191-202. 